\title{
Rehabilitation nach Schlaganfall: Besser mit einem Antidepressivum?
}

\begin{abstract}
In kleineren Studien konnte gezeigt werden, dass Serotonin-Wiederaufnahme-Hemmer (SSRI) die Erholung der motorischen Funktionen nach einem Schlaganfall verbessern können. Dies sollte in der vorliegenden multizentrischen Studie prospektivrandomisiert untersucht werden.
\end{abstract}

- In einer multizentrischen Doppelblindstudie wurden prospektiv Patienten mit einem ischämischen Schlaganfall und einer schweren motorischen Beeinträchtigung (Fugl-Meyer-Punktwert, FFMS $\leq 55$ ) randomisiert im Verhältnis $1: 1$ einer Behandlung mit $20 \mathrm{mg}$ Fluoxetin pro Tag oder Placebo zugeteilt. Der Einschluss sollte fünf bis zehn Tage nach dem Schlaganfall erfolgen. Der primäre Beobachtungsparameter war eine Änderung des FFMS nach 90 Tagen Behandlung.

Insgesamt 118 Patienten wurden in die Studie eingeschlossen. Jeweils $59 \mathrm{~Pa}$ tienten wurden der Fluoxetin- beziehungsweise der Placebobehandlung zugelost. In der finalen Analyse konnten 113 Patienten berücksichtigt werden. Nach 90 Tagen unterschied sich der FMMS signifikant in der mit Fluoxetin behandelten Gruppe (34,0; 95\%-Konfidenzintervall [KI] $29,7-38,4$ vs. 24,3; 95\%-KI 19,9-28,7; p = 0,003). Der An-

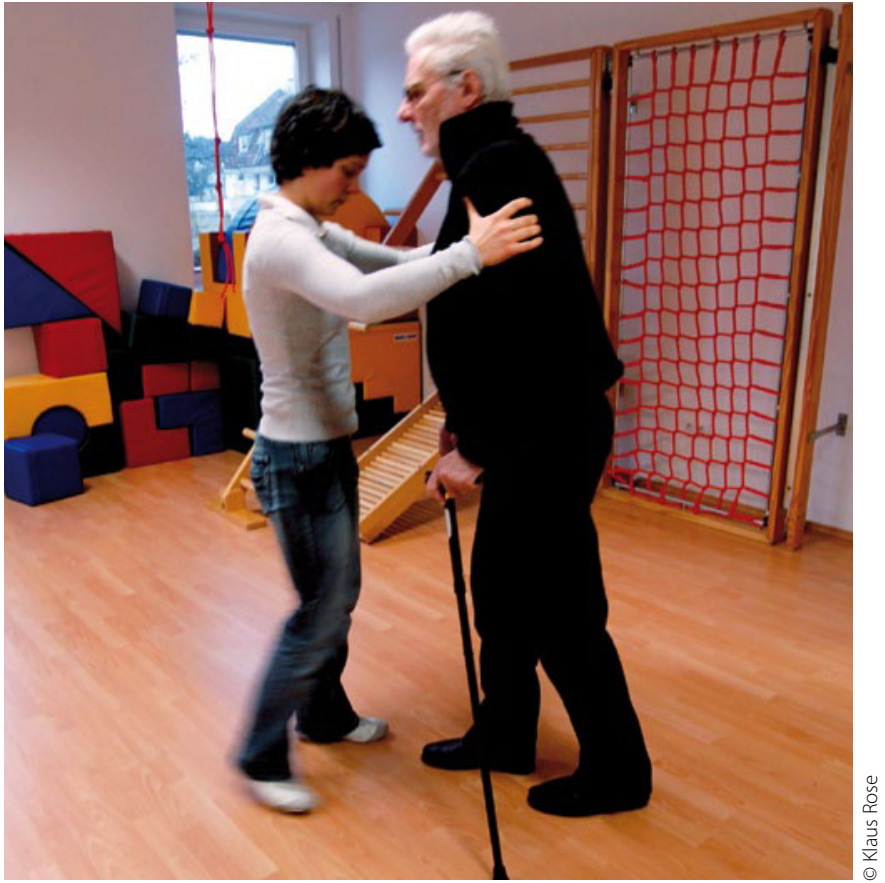

Motorische Ausfälle nach Schlaganfall: Schnelle Besserung mit SSRI.

teil an Patienten mit einer leichten Einschränkung war in der Verumgruppe größer (26\% vs. $9 \%)$ und Depressionen traten seltener auf (7\% vs. $29 \%$ ).

Bei Patienten mit einem ischämischen Schlaganfall mit ausgeprägten motorischen Defiziten verbessert die Fluoxetin die motorische Funktion. Eine positive Beeinflussung des spontanen
Rehabilitationserfolges mit diesem Medikament scheint ein erfolgsversprechender Behandlungsschritt in der Schlaganfalltherapie zu sein.

\section{- F. Chollet et al.}

Fluoxetine for motor recovery after acute ischaemic stroke (FLAME): a randomised placebo-controlled trial. Lancet Neurol 10 (2011) 123-130

\section{Kommentar}

Die hier vorgestellte Studie ist die bisher größte klinische Untersuchung, in der randomisiert der positive Effekt von einem Serotonin-Wiederaufnahme-Hemmer (SSRI) auf die motorische Funktion nach einem Schlaganfall untersucht worden ist. In dieser Studie konnte nicht nur gezeigt werden, dass sich die motorische Funktion nach 90 Tagen verbesserte, sondern auch der Anteil von $\mathrm{Pa}$ tienten mit leichten Einschränkungen (MRS 0-2) war nach dem Beobachtungszeitraum größer in der mit Fluoxetin behandelten Gruppe. Wie zu erwarten war, traten auch depressive Episoden seltener auf.

Da sich nach der Verumbehandlung ein besseres klinisches Outcome zeigte, ist - nach Reproduktion der Ergebnisse - eine Änderung in der medikamentösen Therapie des Schlaganfalles zu erwarten. Dann stünde ein Medikament zur Verfügung, das wegen seines günstigen Nebenwirkungsprofils und der einfachen Anwendbarkeit einer großen Anzahl von Patienten zugänglich gemacht werden könnte. Auch wenn der exakte Mechanismus der SSRIs hinsichtlich der Schlaganfallrehabilitation noch weiter aufzuklären ist, so wird dieser wahrscheinlich einen nicht vaskulären Weg beschreiten. Damit würden diese Medikamente additiv zu den bisherigen Rekanalisationstherapien zur Verfügung stehen.

Die Tatsache, dass bei fast jedem dritten Patienten in der Placebogruppe innerhalb von 90 Tagen eine Depression auftrat, sollte uns schon heute frühzeitig daran denken lassen, diese Medikamente nach einem ischämischen Schlaganfall mit motorischen Defiziten großzügiger anzuwenden.

PD DR. MED. K. GRÖSCHEL, MAINZ . 